\title{
Charm and Beauty Production from Secondary Vertexing at HERA
}

\author{
Paul Thompson ${ }^{* \dagger}$ \\ University of Birmingham, Birmingham B15 2TT, UK \\ E-mail: pdt@hep.ph.bham.ac.uk
}

\begin{abstract}
Measurements of cross sections for events with charm and beauty jets in deep inelastic scattering at HERA are presented. The numbers of charm and beauty jets are determined using variables reconstructed using information from the vertex detectors of the H1 and ZEUS experiments. The jet cross section measurements are compared with QCD predictions and with previous measurements where heavy flavours are identified using muons. The method is also used to measure the heavy flavour proton structure functions $F_{2}^{c \bar{c}}$ and $F_{2}^{b \bar{b}}$.
\end{abstract}

35th International Conference of High Energy Physics - ICHEP2010,

July 22-28, 2010

Paris France

\footnotetext{
${ }^{*}$ Speaker.

† On behalf of the H1 and ZEUS Collaborations
} 


\section{Introduction}

The production of heavy flavour quarks in deep inelastic scattering (DIS) at the HERA electronproton collider is of particular interest for testing calculations in the framework of perturbative quantum chromodynamics (QCD). The process has the special feature of involving two hard scales: the square root of the photon virtuality $Q$ and the heavy quark mass $m$. In the case of jet production the transverse energy $E_{T}$ of the jet provides a further hard scale. In leading order (LO) QCD, the photon-gluon fusion (PGF) processes $e p \rightarrow e c \bar{c} X$ and $e p \rightarrow e b \bar{b} X$ are the dominant production mechanisms for charm and beauty quarks respectively.

The analyses presented here report on measurements by the H1 and ZEUS collaborations of the cross sections for events with charm and beauty jets both in photoproduction [1] and in DIS [2] [3] at HERA. The analyses use secondary vertex flavour tagging to distinguish the jets that contain charm and beauty flavoured hadrons from those containing light flavoured hadrons. The measurements also include the contribution of charm and beauty quarks to the proton structure function $F_{2}^{c \bar{c}}$ and $F_{2}^{b \bar{b}}$ [4].

\section{QCD Models}

The cross section data are compared with the predictions of NLO QCD programs in both photoproduction (FMNR [5]) and DIS (HVQDIS [6]). The programs are based on the PGF $\mathscr{O}\left(\alpha_{s}^{2}\right)$ matrix element and provide weighted events with two or three outgoing partons, i.e. a heavy quark pair and possibly an additional light parton. At NLO the predictions of QCD depend on the choice of the renormalization and factoration scales $\mu_{r}$ and $\mu_{f}$. To investigate the dependence of the predictions on the scales the calculations are repeated varying the values of $\mu_{r}$ and $\mu_{f}$.

\section{Experimental Method}

The H1 and ZEUS collaborations have precise silicon vertex detectors in order to separate beauty, charm and light quark induced events. The separation is performed using the properties of well measured silicon tracks which are associated with the jet axis in the $\eta-\phi$ plane. The jet axis is typically defined as the direction of the jet with the highest $E_{T}^{\mathrm{jet}}$ in the laboratory frame. The impact parameter $\delta$ of a track is the transverse distance of closest approach of the track to the position of the beam interaction region. The sign of $\delta$ for a track is defined with respect to the azimuthal angle of the jet. The track significance $S$ is defined as $S=\delta / \sigma(\delta)$, where $\sigma(\delta)$ is the uncertainty on $\delta$.

The H1 experiment defines $S_{1}, S_{2}$ and $S_{3}$ as the significance of the track with the highest, second highest and third highest absolute significance, respectively. The $S_{1}$ and $S_{2}$ distributions are used for low multiplicity events. For jets with $N_{\text {track }} \geq 3$ an artificial neural network (NN) is used to produce a distribution that combines $S_{1}, S_{2}$ and $S_{3}$ with several variables in order to provide an optimal discrimination between beauty and charm jets. The additional variables include the significance of the transverse distance between the secondary and primary vertex and the number of reconstructed tracks at the secondary vertex. The fractions of events with charm, beauty and light jets in the data are extracted using a least squares simultaneous fit to the $S_{1}, S_{2}$ and NN output distributions, and the total number of events after DIS and jet selection. 

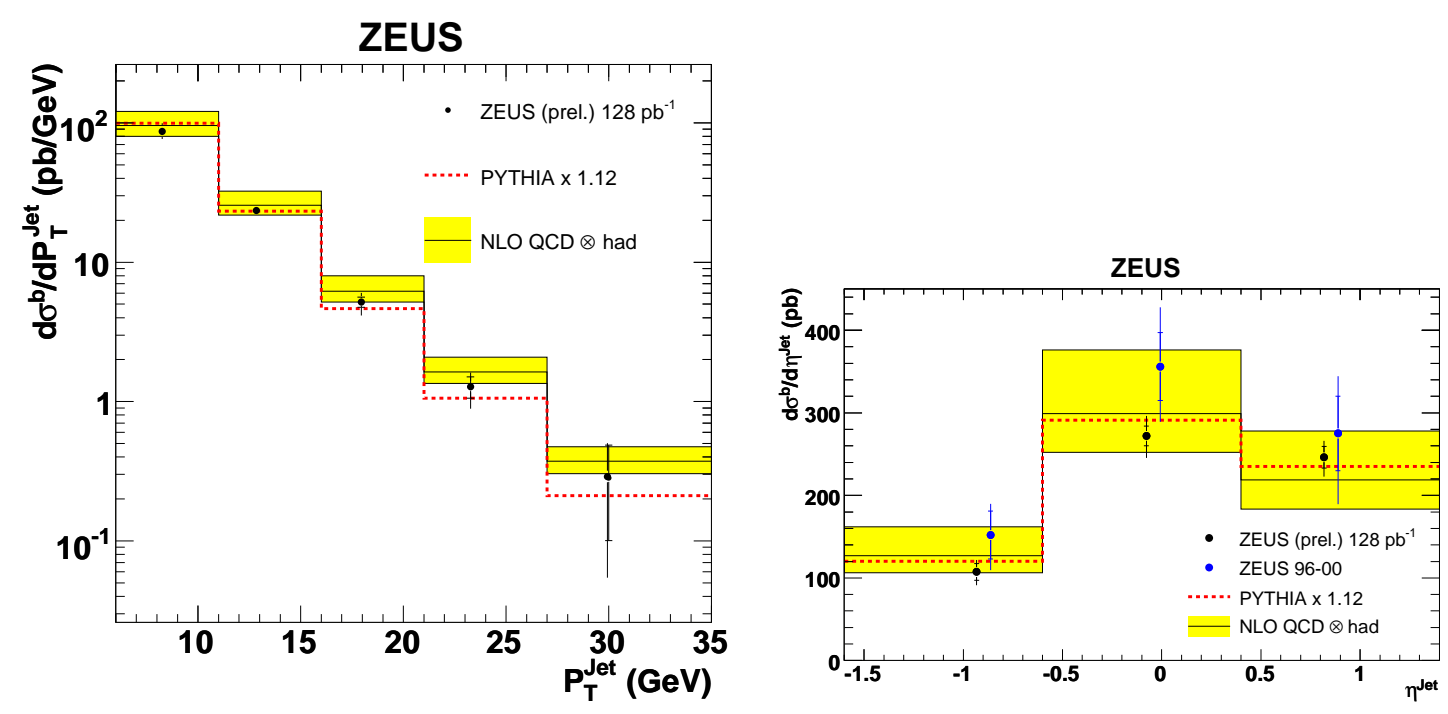

Figure 1: The cross sections for beauty dijet production in photoproduction [1] as a function of $P_{T}^{\text {jet }}$ and $\eta^{\text {jet }}$. The data are compared with the predictions of NLO QCD, the Monte Carlo program PYTHIA and with previous measurements obtained using muons [7].

The ZEUS collaboration uses a similar method and explicitly requires the presence of a reconstructed secondary vertex with a decay length $L_{X Y}$, which is defined as the distance between the beam interaction region and the secondary vertex projected along the jet axis in the $X-Y$ plane. The decay length significance is then formed from $S_{L}=L_{X Y} / \sigma\left(L_{X Y}\right)$, where $\sigma\left(L_{X Y}\right)$ is the uncertainty on $L_{X Y}$. To extract the heavy flavour and light quark fractions a fit is perfomed simultaneously to $S_{L}$ in bins of the invariant mass of tracks from the secondary vertex $M_{\mathrm{VTX}}$. The lower and higher $M_{\mathrm{VTX}}$ regions are sensitive to the charm and beauty contributions respectively.

\section{Results}

\subsection{Beauty Dijets in Photoproduction}

The cross sections for beauty dijets are measured by the ZEUS collaboration in the kinematic range $Q^{2}<1 \mathrm{GeV}^{2}, P_{T}^{\text {jet }}>7(6) \mathrm{GeV}$ and $-1.6<\eta^{\text {jet }}<1.3[1]$. The cross sections as a function of $P_{T}^{\text {jet }}$ and $\eta^{\text {jet }}$ is shown in figure 1 . The cross sections are well described by the PYTHIA Monte Carlo program and also by the predictions of NLO QCD. The cross section as a function of $\eta^{\text {jet }}$ is also compared with the results obtained by muon tagging [7]. Good agreement is found between the two independent methods.

\subsection{Charm and Beauty Jets in DIS}

The cross section for charm and beauty jets has been measured by the H1 collaboration in DIS in the kinematic range $Q^{2}>6 \mathrm{GeV}^{2}$ and $0.07<y<0.625$ for the heavy flavour jet with the highest $E_{T}^{\text {jet }}$ with $E_{T}^{\text {jet }}>6 \mathrm{GeV}$ and $-1.0<\eta^{\text {jet }}<1.5$ [2]. The differential charm jet cross sections are shown as a function of $E_{T}^{\text {jet }}$ and $\eta^{\text {jet }}$ in figure 2. The data are compared with the NLO predictions of HVQDIS including hadronisation corrections. In general the NLO expectations describe the 

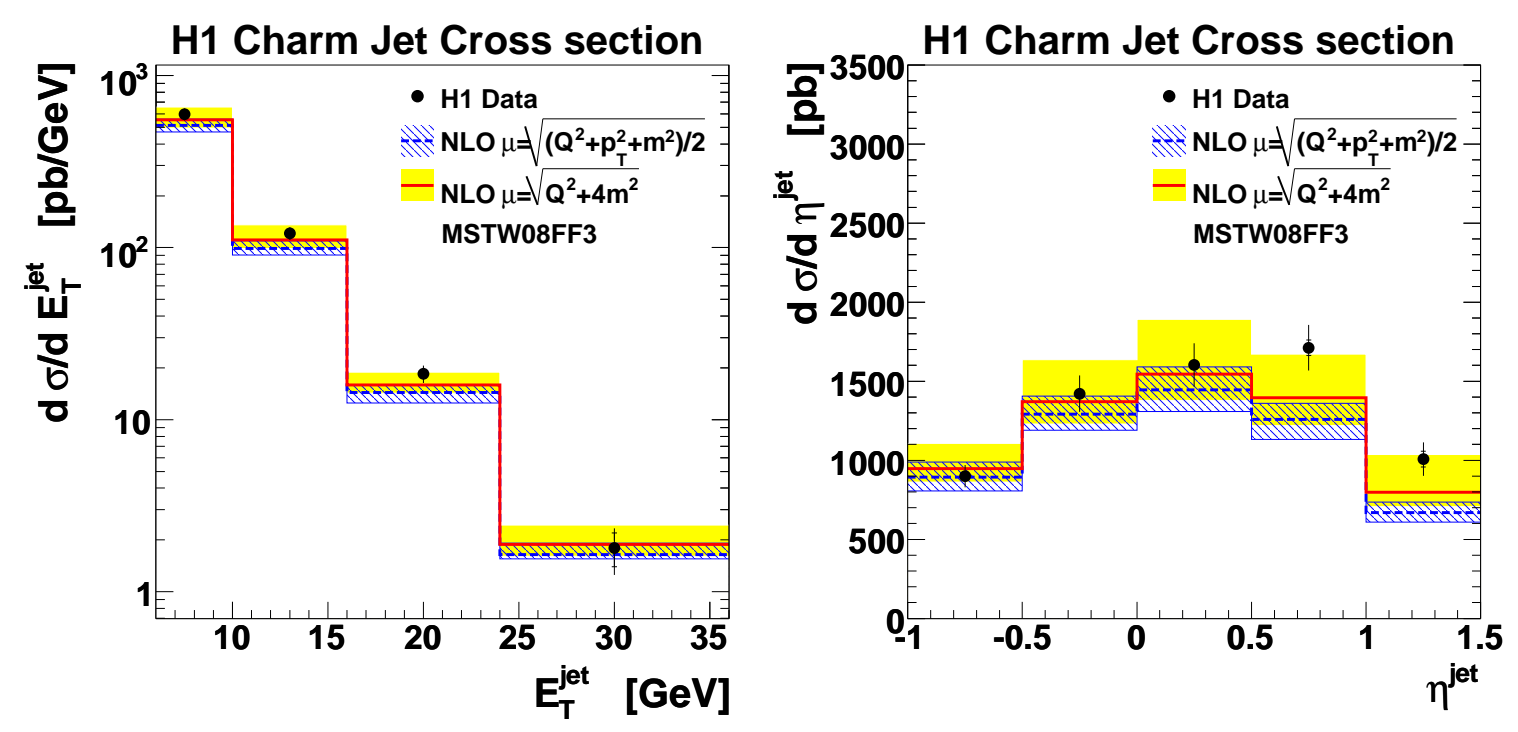

Figure 2: The cross sections for charm jet production as a function of $E_{T}^{\text {jet }}$ and $\eta^{\text {jet }}$ [2]. The predictions of NLO QCD are also shown.

charm data reasonably well in all differential distributions although the predictions with the scale $\mu=\sqrt{\left(Q^{2}+p_{T}^{2}+m^{2}\right) / 2}$ fall somewhat below the data at low $E_{T}^{\text {jet }}$ and in the forward $\eta^{\text {jet }}$ region. The differential beauty jet cross section in the laboratory frame is shown as a function of $E_{T}^{\text {jet }}$ in figure 3 (left). NLO QCD provides a good description of the beauty data.

The cross section for beauty jets has also been measured by the ZEUS colaboration in the kinematic range $5<Q^{2}<1000 \mathrm{GeV}^{2}$ and $0.02<y<0.7$ [3]. Secondary vertices are fitted using jets with $E_{T}^{\text {jet }}>5 \mathrm{GeV}$ and $-1.6<\left|\eta^{\text {jet }}\right|<2.2$. The analysis uses $354 \mathrm{pb}^{-1}$ of integrated luminosity. The beauty jet cross sections are found to be generally well described by the predictions of NLO. For the lowest values of $Q^{2}$ the predictions tend to lie below the data.

$4.3 F_{2}^{c \bar{c}}$ and $F_{2}^{b \bar{b}}$

The technique of secondary vertex tagging can also be used to measure the heavy flavour structure functions $F_{2}^{c \bar{c}}$ and $F_{2}^{b \bar{b}}$. The measurements of $F_{2}^{c \bar{c}}$ obtained using secondary vertex tagging are found to be in good agreement with measurements obtained using exclusive $D$ meson reconstruction. The charm measurements have a similar precision to the differences between the QCD NLO and NNLO predictions. These two independent methods for measuring the charm cross section may be combined to improve the precision of $F_{2}^{c \bar{c}}$ and further test the predictions of QCD.

The measurements of $F_{2}^{b \bar{b}}$ from secondary vertex methods at HERA are shown in figure 3 (right). The results from the two experiments are found to be in good agreement. The data are well described by the predictions of NLO and NNLO QCD. There are differences in the QCD predictions but these are small in comparison with the experimental accuracy of the measurements.

\section{Summary}

The technique of secondary vertex flavour tagging provides a method to measure the properties 

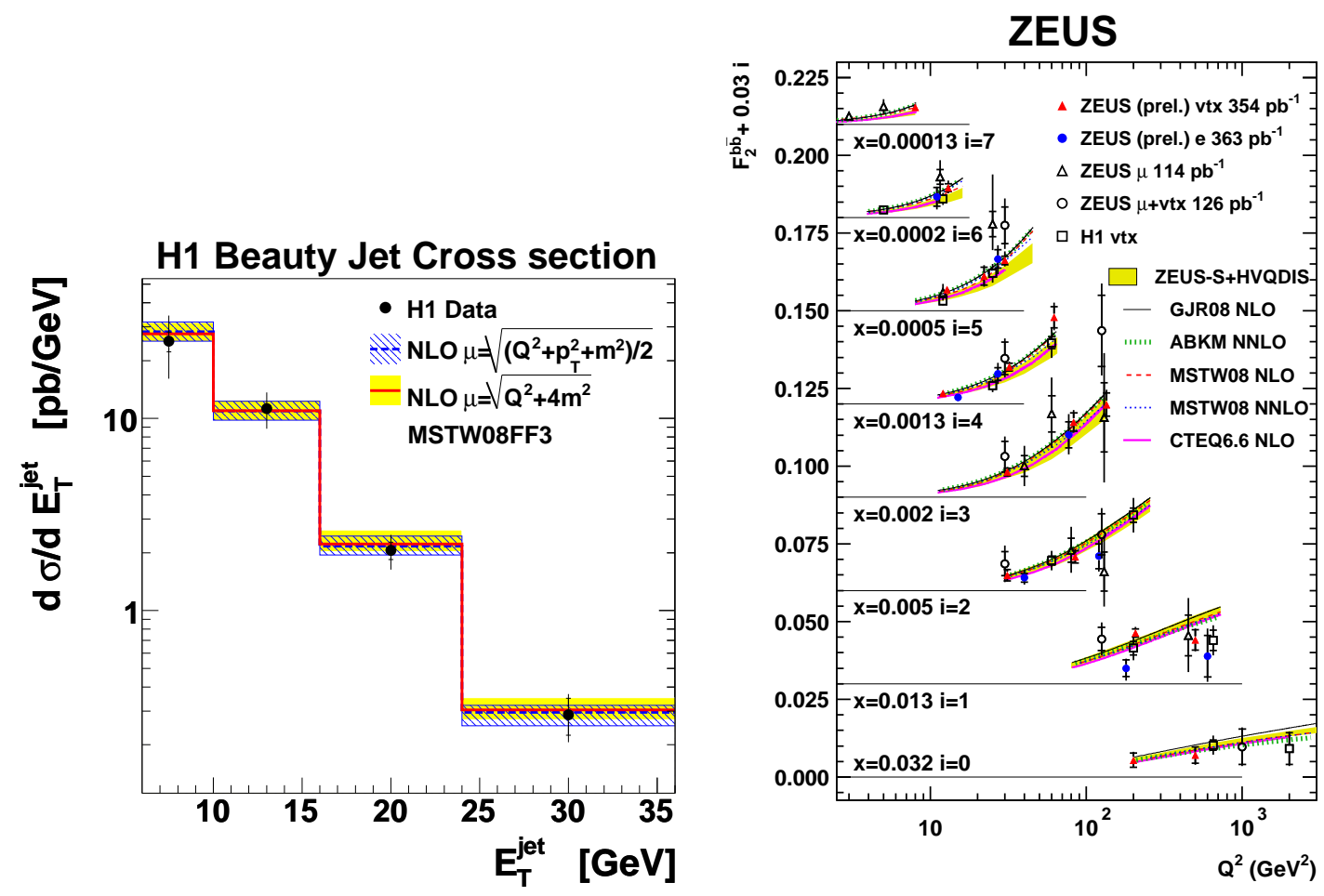

Figure 3: The cross section for beauty jet production [2] as a function of $E_{T}^{\text {jet }}$ (left). The measurements from H1 [4] and ZEUS [3] of the structure function $F_{2}^{b \bar{b}}$ as a function of $Q^{2}$ for fixed $x$ (right). The measurements are compared with the predictions of QCD.

of charm and beauty production at HERA. The present heavy flavour jet measurements show that charm and beauty production in deep inelastic scattering is adequately described by NLO QCD in the presence of an additional hard scale provided by a jet.

\section{References}

[1] [ZEUS Collaboration], ZEUS Preliminary ZEUS-prel-09-005.

[2] F. D. Aaron et al. [H1 Collaboration], [arXiv:1008.1731].

[3] [ZEUS Collaboration], ZEUS Preliminary ZEUS-prel-10-004.

[4] F. D. Aaron et al. [H1 Collaboration], Eur. Phys. J. C 65 (2010) 89 [arXiv:0907.2643].

[5] S. Frixione, M. L. Mangano, P. Nason and G. Ridolfi, Phys. Lett. B 348 (1995) 633 [hep-ph/9412348].

[6] B. W. Harris and J. Smith, Phys. Rev. D 57 (1998) 2806 [hep-ph/9706334];

B. W. Harris and J. Smith, Nucl. Phys. B 452 (1995) 109;

B. W. Harris and J. Smith, Phys. Lett. B 353 (1995) 535.

[7] S. Chekanov et al. [ZEUS Collaboration], Phys. Rev. D 70 (2004) 012008 [Erratum-ibid. D 74 (2006) 059906] [hep-ex/0312057]. 